\title{
Correlation between the maximum wavelength and the line width in the emission of a single semiconductor colloidal quantum dot at different temperatures
}

\author{
Eduard Podshivaylov ${ }^{1}$, Maria Kniazeva ${ }^{1}$, Ivan Eremchev ${ }^{2}$, Andrey Naumov ${ }^{2,3}$, and Pavel \\ Frantsuzov ${ }^{1, *}$ \\ ${ }^{1}$ Lomonosov Moscow State University, 119991 Moscow, Russia \\ ${ }^{2}$ Institute of Spectroscopy RAS, 108840 Moscow, Russia \\ ${ }^{3}$ Moscow State Pedagogical University, 119435 Moscow, Russia
}

The very first measurements [1] of the spectrally resolved photoluminescence of single colloidal semiconductor quantum dots CdSe (QDs) revealed the phenomenon of very slow spectral diffusion (SD) with a characteristic time scale in order of hundred seconds. Müller et al. [2,3] found correlation between linewidth and peak position of the single QD spectrum at temperatures from $5 \mathrm{~K}$ to $300 \mathrm{~K}$. Usual explanation of this phenomenon [2-4] is based on the quantum-confined Stark effect by the movement of charge carriers around individual QDs. If this hypothesis is correct, the effect should depend on the dielectric permittivity of the media. Gomez et al. [5] found no dependence on the dielectric permittivity.

A unique spectroscopic experiment on single QD under slow heating and cooling was carried out by our group. Fluorescence images and spectra of single quantum dots were measured via home-build fluorescence microscope equipped by prism spectrometer. Two optical schemes: wide-field and scanning confocal schemes were combined in one microscope in order to simplify procedure of single quantum dots preliminary searching (by fluorescence image processing and antibunching determination) and sequential measuring of fluorescence spectra of the selected QD.

Quantum dots (CdSe/ZnS, Sigma Aldrich with Fluorescence peak at $620 \mathrm{~nm}$ ) were dispersed in toluene solution of polyisobutylene in low concentration and then spincoated onto a cover glass. Thicknesses of polymer films with single quantum dots were varied in the range of several tens of nanometers. Sample temperature was controlled by LakeShore temperature controller and regulated by Peltier element attached to the sample. The experiment presented here was carried out at ambient conditions.

Tunable dye laser (Coherent CR599) was used to excite quantum dots at the wavelength of $580 \mathrm{~nm}$ near the quantum dot absorption band edge. Excitation laser intensity $\left(\sim 100 \mathrm{~W} / \mathrm{cm}^{2}\right.$ in a focused spot) was attenuated by neutral spectral density filters (Standa) and controlled by Newport Power meter. Set of interference filters separated fluorescence

\footnotetext{
*Corresponding author: pavel.frantsuzov@gmail.com
} 
signal from scattered laser radiation. Two highly sensitive cooled EMCCD cameras were utilized to capture single quantum dot images and spectra. Hanbury Brown and Twiss scheme with broadband 50\% splitter and two identical SPAD detectors with time resolution $1 \mathrm{~ns}$ and dead time $200 \mathrm{~ns}$ (QE $50 \%$ ) were used to measure autocorrelation function for QD fluorescence intensity. Some details of the apparatus and measurements procedures were described previously in [6,7].

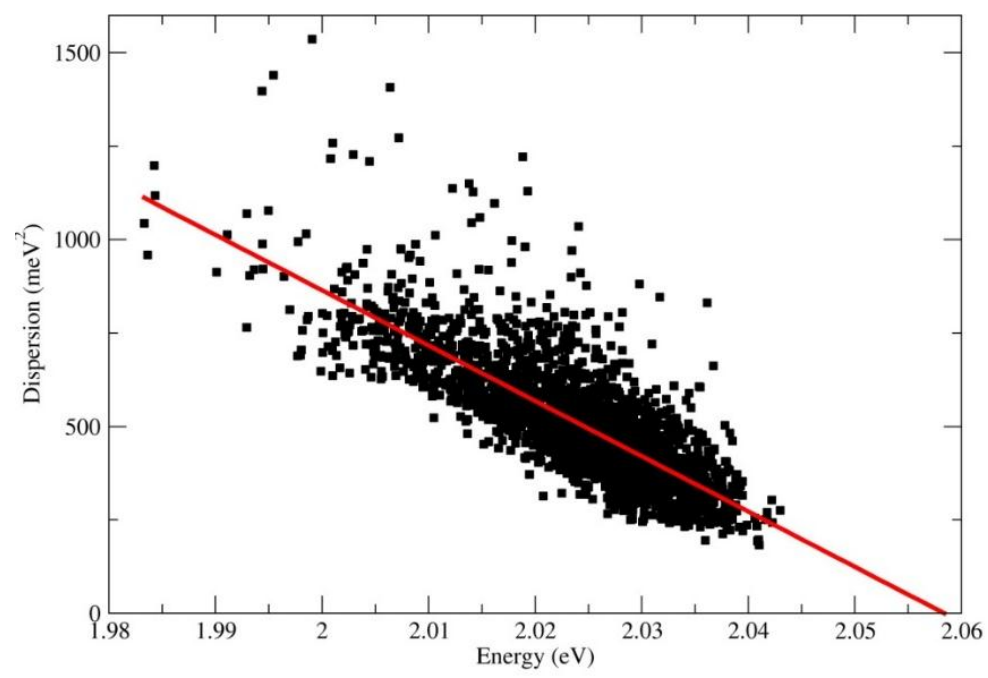

Fig. 1. The dependence of the dispersion on the peak wavelength. The points correspond to the experimentally obtained values at room temperature. The solid line indicates the theoretical dependence obtained in the proposed model based on the fluctuations of the electron-phonon interaction.

Fluorescence spectra sequences for single quantum dots were measured with exposure time $200 \mathrm{~ms}$ and spectral resolution $\sim 0.7 \mathrm{~nm}$, which was enough to rich good signal to noise ratio.

The experiments show a correlation between the position of the maximum and width of the QD emission spectrum (see Fig. 1). In order to explain experimental results we propose a new model of the QD spectral diffusion and temperature dependent shift. In the model both the peak position and the linewidth are determined by the electron-phonon interaction value. Slow variations of this value in time lead to correlated fluctuations of the wavelength and linewidth at given temperature.

The study was supported by the Russian Foundation for Basic Research, project 16-02-00713.

\section{References}

1. S. A. Empedocles, D. J. Norris, and M. G. Bawendi, Phys. Rev. Lett. 77, 3873 (1996)

2. S. A. Empedocles and M. G. Bawendi, Science, 278, 2114 (1997)

3. J. Muller, J. M. Lupton, A. L. Rogach, J. Feldmann, D.V. Talapin, and H. Weller, Phys. Rev. Lett., 93, 167402 (2004)

4. J. Muller, J. M. Lupton, A. L. Rogach, J. Feldmann, D.V. Talapin, and H. Weller, Phys. Rev. B, 72, 205339 (2005)

5. D. E. Gómez, J. van Embden, and P. Mulvaney, Appl. Phys. Lett., 88, 154106 (2006)

6. I. Y. Eremchev, I. S. Osad'ko, A. V. Naumov, J. Phys. Chem. C, 120, 22004 (2016)

7. I. S. Osad'ko, I. Y. Eremchev, A. V. Naumov, J. Phys. Chem. C, 119, 22646 (2015) 